\title{
CONSTRUÇÃO DE CONHECIMENTO EM AMBIENTE DIGITAL: A IMPORTÂNCIA DA PERSPECTIVA DIALÓGICA
}

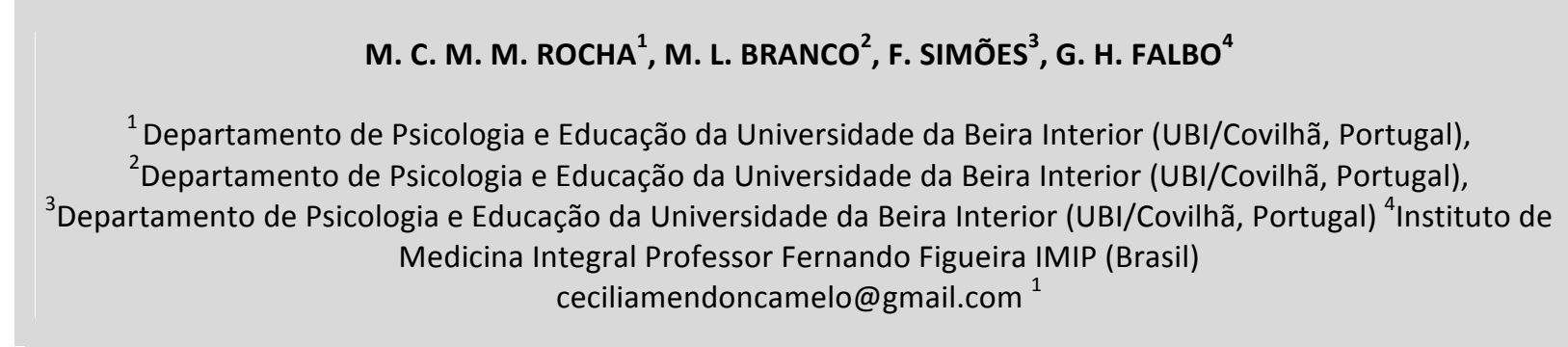

Submetido 31/10/2016 - Aceito 16/10/2018

DOI: $10.15628 /$ holos.2018.4559

\section{RESUMO}

Este artigo tem como objetivo apresentar alguns pressupostos teóricos e discutir os aspectos conceituais da Aprendizagem Baseada em Problemas (ABP) e suas contribuições para o ensino superior, trazendo também uma breve reflexão sobre o cenário imperativo tecnológico. Este escopo teórico está sendo desenvolvido dentro de uma pesquisa de doutorado em Educação da Universidade da Beira Interior (Covilhã,
Portugal), cujo objetivo é identificar as estratégias de aprendizagem utilizadas pelos alunos do 5o ano de uma escola de medicina de uma instituição de ensino superior em saúde, localizada no nordeste do Brasil, em um curso complementar sobre segurança e qualidade na atenção ao paciente oferecido em uma plataforma digital.

PALAVRAS-CHAVE: Conhecimento, Educação a Distância, Educação móvel, Aprendizagem baseada em problemas, Educação médica.

\section{KNOWLEDGE CONSTRUCTION IN A DIGITAL ENVIRONMENT : The importance of dialogical perspective}

\begin{abstract}
This article aims to present some theoretical assumptions and discuss the conceptual aspects of Problem Based Learning (PBL) and its contributions for higher education, also bringing a brief reflection on the technological imperative scenario. This theoretical scope is being developed within a doctoral research in Education at the University of Beira Interior (Covilhã,
\end{abstract}

Portugal) whose goal is to identify the learning strategies experienced by students of the 5 th year of a medical school of a higher health educational institution, located in northeastern Brazil in a complementary course on Patient Care offered on a digital platform. 


\section{INTRODUÇÃO}

Refletir sobre as mudanças pedagógicas que vêm sendo implementadas nas formas de ensinar e aprender provoca a busca pelo entendimento da importância da adoção de propostas de metodologias ativas que aportem os processos de aprendizagem. A Aprendizagem Baseada em Problemas (ABP) é uma das ferramentas utilizadas para modificar os conceitos do ensino tradicional (Mezzari, 2011) e seu método se caracteriza pela forte motivação prática e estímulo cognitivo com o intuito de solucionar problemas. O processo de aprendizagem surge de situações problemas com o objetivo de provocar dúvidas que estimulem desafios intelectuais aos estudantes (Mezzari, 2011; Cyrino \& Toralles-Pereira, 2004). Nesta proposta metodológica a sala de aula deve constituir-se em um espaço coletivo de construção de conhecimento; um lugar de formação de pessoas capazes de serem autônomas, cientes de suas vidas e de suas escolhas (Borochovicius \& Tortella, 2014). Pretende-se que, neste cenário, a aprendizagem se construa de forma compartilhada: todos envolvidos em um processo de construção de conhecimento onde os sujeitos não podem mais não se comprometer e ser apenas um mero espectador (Cyrino \& Toralles-Pereira, 2004; Borochovicius \& Tortella, 2014; Dewey, 2004).

As propostas de metodologias ativas como a ABP despontam como uma estratégia; uma proposta de reestruturação curricular que objetiva a integração de contextos tendo em vista a prática e a solução de problemas; com destaque para a utilização das tecnologias e suas ferramentas de interação e hipermídia, buscando integrar ao tempo de sala de aula atividades em ambientes virtuais que possibilitem discussões para fixação da aprendizagem de forma compartilhada (Kuri, Manzato \& Silva, 2004; Cyrino \& Toralles-Pereira, 2004). Neste contexto de mudanças e inquietudes, as escolas precisam se adaptar a um novo padrão de aluno a quem é solicitado que não assuma uma posição de passividade diante da sua aprendizagem. E na tentativa de acolher um novo perfil de estudantes que chega aos cursos de graduação, as instituições de ensino superior procuram atender às demandas dessa nova geração de estudantes por meio da adoção de metodologias e novas propostas pedagógicas que assegurem a qualidade e a efetividade do ensino. Como motivação para agregar este perfil de estudantes uma possibilidade é a utilização de métodos pedagógicos que permitam ao estudante desempenhar um papel mais ativo, garantindo-lhe maior autonomia no processo de aprendizagem numa sociedade inserida no cenário de imperativo tecnológico que vivenciamos (Borochovicius \& Tortella, 2014; Soares, 2008).

Nesta ótica, buscamos entender qual a relevância de tais mudanças no processo de construção de conhecimento dos sujeitos onde estes deixam de ser ouvintes passivos - conforme previam as propostas tradicionais de ensino - e passam a assumir posturas ativas, comprometidas com seu processo de aprendizagem com a tecnologia cada vez mais presente no cenário educacional. Diante destes novos contextos, as instituições de ensino precisam estar abertas às mudanças e ao impacto digital onde o "desafio maior é caminhar para um ensino e uma educação de qualidade, que integre todas as dimensões do ser humano" (Moran, Masetto \& Behrens, 2000, p. 15). 
Neste artigo apresentamos alguns pressupostos teóricos da nossa pesquisa, discutimos os aspectos conceituais da ABP e seus subsídios para o ensino superior, especificamente para o ensino médico, e fazemos também uma breve reflexão sobre o cenário de imperativo tecnológico atual. Este escopo teórico está sendo desenvolvido na pesquisa de doutorado com o projeto de tese Repensando novas estratégias e cenários para a construção de conhecimento que tem por objetivo identificar as estratégias de construção de conhecimento dos estudantes da graduação de um curso de medicina em uma plataforma digital. Procuramos também identificar como os estudantes concebem o seu processo de aprendizagem: se o compreendem como um simples processo de transmissão de informação - como previa a proposta pedagógica em um modelo tradicional - ou se já evidenciam o entendimento sobre a imprescindível participação ativa do educando na construção do seu conhecimento, conforme a proposta pedagógica da Aprendizagem Baseada em Problemas (ABP) adotada pela Instituição de Ensino Superior (IES) cenário desta investigação. Acreditamos que os resultados desta pesquisa possam contribuir no aprofundamento da percepção sobre a construção de conhecimento com autonomia; assim como na proposição de diretrizes que possibilitem a estruturação de módulos em plataformas digitais sintonizados com a proposta pedagógica da ABP.

\section{A APRENDIZAGEM BASEADA EM PROBLEMAS (ABP)}

A prática construtivista coloca o professor como motivador do raciocínio dos alunos e tem como objetivo provocar conflitos cognitivos; gerar dúvidas em relação ao objeto do conhecimento com a função de, através de um processo de interação entre os sujeitos, possibilitar a ampliação da estrutura cognitiva mediante uma aprendizagem ativa. Contudo, para que a aprendizagem aconteça ela precisa transformar, transcender e exigir apreensão de novos significados, relacionando-os às experiências prévias e às vivências dos alunos, permitindo a formulação de problemas que estimulem, desafiem e incentivem novas aprendizagens. Nesse contexto, a $\mathrm{ABP}$, proposta com forte estímulo ao pensamento crítico, surge na intenção de auxiliar o discente no processo de construção de conhecimento, fortalecer a sua capacidade de resolver problemas e envolvê-lo no aprendizado. A ABP tem como axioma o uso de problemas da vida real para instigar o desenvolvimento conceitual, procedimental e atitudinal do discente (Borochovicius \& Tortella, 2014). Nesta proposta pedagógica o conhecimento deve se iniciar por um problema e se encerrar com a resolução dele (Dewey, 2004). De acordo com Penaforte (2001, apud Cyrino \& Toralles-Pereira, 2004), a ABP se fundamenta na teoria do conhecimento do filósofo pragmatista americano John Dewey e sua base intelectual está situada nos pressupostos de Dewey sobre aprendizagem autônoma e utilização de problemas que antecedem a formação de conceitos, ressaltando a importância de aprender a partir de uma situação real. É uma proposta que rompe com o modelo tradicional do ensino e da aprendizagem. De acordo com Cyrino e Toralles - Pereira (2004, p.782)

a aprendizagem parte de situações que intencionam gerar dúvidas, o método valoriza experiências concretas e problematizadoras, com forte motivação prática e estímulo cognitivo para solicitar escolhas e soluções criativas por parte do educando ... estimula processos de interações ativas que levem o aluno a uma aprendizagem significativa. 
Na esfera da educação médica a ABP surgiu na década de 60 do século $X X$ quando foi institucionalizada a partir da experiência na Universidade de McMaster/Canadá e, posteriormente, seguida na Universidade de Maastricht / Holanda (Melo, 2012, p.45). Partiu de uma iniciativa da Universidade de McMaster / Canadá de tentar modificar a forma como a medicina vinha sendo ensinada, e esta mudança de proposta pedagógica possibilitaria que os estudantes de medicina ampliassem suas habilidades para resolver problemas avaliando, interpretando e aplicando "informações que trouxessem melhores respostas aos pacientes" (Borochovicius \& Tortella, 2004, p.267).

\section{Cenários Ativos e Construção de Conhecimento}

O compartilhamento de ideias através de discussões que estimulam a construção do conhecimento de forma colaborativa mediante ações comunicativas e dialógicas tem como facilitador, neste processo, o docente desempenhando um papel de maestro: regendo os diálogos. Na sua proposição que distingue discurso e ação Habermas esclarece que é apenas "com a transição da ação para o discurso que os participantes adotam uma atitude reflexiva e, à luz de razões pró e contra apresentadas, disputam pela verdade tematizada de enunciados controversos" (Habermas, 2004, p.49). No processo de construção de conhecimento às trocas, através da comunicação e do diálogo, pressupõem, de acordo com Vigotsky,

a transmissão racional e intencional de experiências e pensamento entre os sujeitos e requer um sistema mediador, cujo protótipo é a fala humana ... A linguagem é o sistema simbólico básico de todos os grupos humanos e fornece os conceitos e as formas de organização do real que constituem a mediação entre o sujeito e o objeto de conhecimento (Vigotsky, 2008, p.8, 70).

Entendemos que quando se exclui "a linguagem e qualquer outro meio de intercomunicação, o desenvolvimento intelectual dos seres humanos ... torna-se impossível" (Dewey, 2004, p. 211). Diante disto, buscamos entender o processo de construção de conhecimento que surge prioritariamente através das relações comunicativas e das interações partilhadas pelos sujeitos. E seguimos como orientação a ideia que há maior probabilidade de construção de um conhecimento mais consistente por parte dos estudantes quando existe uma ação docente mediadora e comunicativa de estímulo à utilização reflexiva e autônoma dos recursos disponíveis, sejam estes tecnológicos ou não, assim como adoção de uma postura dialógica entre os sujeitos. No contexto tecnológico, partimos de uma pergunta norteadora: em um ambiente virtual os estudantes são capazes de desenvolver e gerir com autonomia seu processo de aprendizagem, estruturando estratégias que estimulem a atingir satisfatoriamente os objetivos propostos? Pois, neste cenário ativo, salientamos a importância que sejam estruturadas estratégias, por parte dos discentes, que visem a otimização da utilização das informações disponíveis. Portanto, entendemos que as propostas educacionais que orientam o processo de ensino e aprendizagem neste início de século devem ter como base não apenas a capacitação dos sujeitos, mas a completa realização do potencial humano. E a inserção das tecnologias nesse processo deve ser entendida como algo que está além do seu uso. Está no 
entendimento da lógica que passa através da movimentação entre os saberes no atual estágio da sociedade tecnológica (Delors, 1998; Kenski, 2003; Melo, 2012).

\subsection{Contextos Digitais e Estratégias Discentes}

Alguns estudos na área da educação demonstram que as formas tradicionais de ensino de repasse de conteúdos, centradas no professor, já não dão conta de atender as mudanças que ocorrem nas sociedades e nos contextos educacionais (Mezzari, 2011; Cyrino, Toralles-Pereira, 2004); tornando evidente que uma das competências mais relevantes a ser desenvolvida pelo estudante - e fundamental para solução de problemas - é a capacidade de desenvolver habilidades em buscar a informação, bem como saber selecioná-la e utilizá-la de forma crítica.

A inserção da tecnologia no processo de construção de conhecimento reestrutura as relações com o saber e ampliam as possibilidades de se integrar, de se comunicar e trocar informações instantaneamente (Moran, 2000; Melo, 2012; Lévy, 2003). No entanto, para que os recursos tecnológicos não se configurem em simples aparato, é imprescindível entender sua significação além do mero acesso. Estabelecendo situações para que o estudante possa se expressar através das múltiplas linguagens, domine a funcionalidade das tecnologias e desenvolva habilidades específicas na compreensão de suas propriedades e suas potencialidades, na busca da solução de problemas (Moran, 2000; Almeida, 2003). A desconstrução das barreiras do tempo e do espaço, proporcionada pelas tecnologias, viabiliza o trabalho colaborativo e a troca de informações em tempo real (Lévy, 2003; Moran, 2000; Melo, 2012). Impulsionando o trabalho em rede e o processo de construção de conhecimento, pois, a criatividade, a autonomia e a capacidade de autoprogramação dos trabalhadores do conhecimento não seriam produtivas se não pudessem combinar-se com o trabalho em rede (Castells, 2013, p. 65).

As propriedades dos cenários com a inclusão das tecnologias "possibilitam ao utilizador a experimentação de certo grau de autonomia enquanto navega na informação ... contribuindo para que se expressem estratégias individuais de aprendizagem, sendo o sujeito responsável pelo seu próprio processo de aprendizagem" (Ferreira, 2008, p. 177), aprendendo a pensar suas estratégias e possibilidades de construir conhecimento. Nos cenários digitais, os estudantes têm a autonomia para autorregular seu processo de aprendizagem fazendo suas opções de ações e adotando estratégias que podem enriquecer o processo de aprendizagem e, assim, alcançar melhor seus objetivos. Fazendo um contraponto com o princípio de Dewey (2004, p. 49) sobre a autonomia do "aprender a aprender", proposto pelas metodologias ativas, como a ABP, na proposição sobre trabalhadores auto programáveis e trabalhadores genéricos como a divisão fundamental numa sociedade em rede, ponderamos que

os trabalhadores auto programáveis têm a capacidade autónoma de se centrar numa meta que Ihes tenha sido atribuída no processo de produção e encontrar a informação relevante, recombiná-la em forma de conhecimento. ... Esta capacidade de procurar recombinar a informação .... requer uma educação adequada, não em termos de habilitação, mas, sim em termos de capacidade criativa e recursos para evoluir com as mudanças tecnológicas e os novos conhecimentos (Castells, 2013, p. 65). 
Esta maior capacidade de abstração e generalização, de recombinar, reagrupar conceitos e aplicá-los em contextos diferenciados só é possível quando se atinge o que Vigotsky (2008) denomina de pensamento conceitual, contrapondo-se ao pensamento associacionista que somente aplica o conceito aprendido relacionando-o a uma representação: a um caso específico. No pensamento associacionista a capacidade de abstração é frágil, o conceito é sempre vinculado à situações específicas. De acordo com Schwartz (2008, p.78), aprender pressupõe movimentos que alternam entre ruptura e continuidade em relação ao conhecimento anterior e sua articulação com a realidade. $\mathrm{O}$ que significa que o estudante reorganiza e reconstrói a sua forma de pensar sobre um determinado objeto de conhecimento, incorporando novos elementos que o habilitem a responder e a elaborar dúvidas mais complexas. De acordo com Vigostky (2000) o cérebro é um sistema plástico e aberto, que vai se moldando no decorrer do desenvolvimento ontogenético e no desenvolvimento das funções mentais superiores; e consequentemente, no desenvolvimento da sua capacidade de abstração e de pensar o que não estar presente. Neste sentido, podemos trazer a reflexão de que o processo de construção de conhecimento, assim como o ser humano, é inacabado, inconcluso, não estático. Está em construção permanentemente (Freire, 1996). O processo de construção do conhecimento não começa do nada, do vazio. Ao contrário, sempre existe algo que está constantemente em mudança, ou seja; trata-se de um conhecimento que se transforma e se substitui (Vigotsky, 2008), onde se buscam caminhos, entendimentos e novos significados: buscam-se e aliam-se novas estratégias para resolução de problemas.

Estratégia é a "arte de conceber operações de guerra em planos de conjunto" (Melo, 2012, p. 78). No que tange ao cenário da educação, do ponto de vista do aluno, alguns autores entendem que estratégias são procedimentos ou atividades que possibilitam a realização de tarefas. São ações intelectuais conscientes e intencionais, com as quais o aluno se envolve durante a aprendizagem e que podem promover a recuperação de conhecimentos já adquiridos (prévios), enriquecendo a qualidade do processo (Perassinoto, Boruchovitch \& Bzuneck, 2013). Neste sentido, podemos explicitar que estratégias de aprendizagem são os caminhos escolhidos pelo estudante na intenção de potencializar o processo de aprendizagem através das reconstruções dos significados. Estas reconstruções, desenvolvidas a partir da interlocução entre um conhecimento inicial não elaborado e um conhecimento a ser construído através da ação conjunta dos educandos, favorecem o desenvolvimento de "ações cognitivas: observação, confrontação, elaboração de hipóteses, solução de problemas, realizadas pelo aluno ao aprender" (Beluce \& Oliveira, 2012, p. 2). Corroboramos, neste estudo, com os pensamentos de autores (Anastasiou, 2007; Melo, 2012; Tostes, 2011; Beluce \& Oliveira, 2012) que entendem estratégia de aprendizagem como uma sequência de comportamentos realizados pelo estudante para alcançar um objetivo de aprendizagem. Os fatores que caracterizam "as ações constituintes das estratégias" são o planejamento e a intencionalidade (Beluce \& Oliveira, 2012, p. 4), e cabe ao estudante compreender em que momento tais estratégias poderão contribuir significativamente no seu processo de aprendizagem (Anastasiou, 2007; Beluce \& Oliveira, 2012, 2012). As estratégias de aprendizagem podem ser categorizadas como cognitivas e metacognitivas (Anastasiou, 2007; Melo, 2012; Tostes, 2011; Beluce \& Oliveira, 2012), e de 
acordo com Dembo (1994 apud Beluce \& Oliveira, 2012, p.4) as estratégias cognitivas estão relacionadas à construção e ao desenvolvimento dos processos intelectuais e agem diretamente na organização, no armazenamento e no processamento das informações. E as estratégias metacognitivas são as ações conscientes e autorreguladas que permitem ao aluno avaliar o seu próprio pensamento e refletir sobre ele, permitindo o autoconhecimento e a aprendizagem dos conteúdos (Beluce \& Oliveira, 2012). Em estudos anteriores Melo (2012) e Beluce e Oliveira (2012) sistematizaram as estratégias em categorias: as estratégias comunicativas ou de interatividade são referentes a processos cognitivos que têm por base os diálogos que culminam em uma ação comunicativa (Ferreira Filho et al, 2008; Habermas, 2004); as estratégias cognitivas ou de aprendizagem são referentes a processos cognitivos dependentes de relações interpessoais e da mediação por pessoas ou objetos, permitindo o estabelecimento de significados a partir do contexto sociocultural (Dewey, 2004; Habermas, 2004; Vigostky, 2008); e as estratégias metacognitivas são referentes a processos cognitivos conscientes e autorregulados que, sendo mais elaborados, permitem refletir sobre o processo de pensamento em curso e a reformulação dos conceitos (Ferreira Filho et al, 2002; Anastasiou, 2007).

$\mathrm{Na}$ realidade atual, nos contextos de ensino e aprendizagem, já não é mais necessário estar-se presente fisicamente para trocar informações, compartilhar e construir conhecimento. A habilidade de lidar com símbolos que substituem o real é que permite o homem emancipar-se das barreiras do espaço e do tempo presentes (Vigotsky, 2000, p. 54). Como afirma Melo (2012, p. 55) é possível "Hipertextualizar o pensamento!" trazendo novamente a discussão de Vigostky $(2008 ; 2000)$ sobre as funções mentais superiores e a plasticidade do cérebro possibilitando ao indivíduo elaborar e refinar sua capacidade de abstração e generalização, levando-o a construções mais complexas. Os ambientes virtuais possibilitam interação a partir da estruturação de cenários que viabilizam a dinamização do processo de aprendizagem. Entretanto, para que a interação, dinamização e motivação ocorram é imperativo que tais ambientes de aprendizagem sejam estruturados por profissionais que tenham 0 total conhecimento da proposta pedagógica do curso ofertado, capazes de desenvolver e estimular novas "abordagens e habilidades a fim de criar um processo de aprendizagem gerador de autonomia" (Palloff \& Pratt, 2002, p. 28). As novas tecnologias promovem o acesso à informação auxiliando na adoção de novas condutas ofertando recursos didáticos que possibilitam formar um profissional que tenha habilidade em utilizar adequadamente ferramentas tecnológicas e seja capacitado e habilitado a desempenhar sua profissão, a tomar decisões e solucionar problemas. No contexto da educação online, estudos apontam que alunos que obtêm bons resultados possuem características semelhantes: têm autonomia para buscar novas formas de aprender, são motivados e disciplinados, podem apresentar uma atitude mais séria com relação as suas expectativas profissionais e com relação ao curso (Palloff \& Pratt, 2002). No entanto, "são necessárias teorias, estratégias e interação adequadas ... à medida que se planeja o ambiente, é importante que exista uma preocupação tanto computacional como em torno dos pressupostos psicológicos e pedagógicos" (Andrade \& Vicari, 2011, p. 257). Tais ambientes podem oferecer uma nova promessa educacional que permite tanto as condições de ensino concebido totalmente à distância como aquelas realizadas no ensino presencial, implementando e 
ampliando as ações desta prática educativa (Almeida, 2003; Tostes, 2011). Entretanto, no desenvolvimento das ações de "comunicação voltadas a criar ambientes mais propícios e convergentes aos processos de ensino e aprendizagem, destaca-se a importância das tecnologias que devem ser desenvolvidas dentro de um projeto pedagógico personalizado e inovador" (Moran, 1995 apud Schalch \& Melo, 2015, p. 35).

Embora algumas pesquisas apontem que ainda se mostra incipiente o estudo das estratégias discentes utilizadas em ambientes virtuais de aprendizagem, outras demonstram preocupação com a transposição de práticas do ensino tradicional para o ambiente online (Mehlecke, 2006; Melo, 2012; Beluce \& Oliveira, 2012); o que torna imperativo não somente o entendimento do significado do pedagógico nas práticas docentes inseridas no contexto de metodologias ativas, assim como qual a dimensão EAD na prática docente. Neste prisma, a identificação das estratégias discentes utilizadas no ambiente virtual, aliado às novas propostas de práticas docentes, pode viabilizar a estruturação de cursos online que efetivamente alcancem seus objetivos educacionais e que primem pela construção de novos conhecimentos e pela promoção da compreensão e da utilização de estratégias de aprendizagem realizadas pelo aluno para e no desenvolvimento de sua aprendizagem (Alves, 2009).

\subsection{Propondo Contextos de Aprendizagem: Plataformas Digitais}

Com a necessidade de se modernizar e atender à demanda do seu novo perfil de estudantes, as instituições de ensino promovem cursos estruturados em ambientes virtuais. Os ambientes virtuais podem possibilitar interação a partir da estruturação de cenários que viabilizam a dinamização do processo de aprendizagem. Ratificamos o pensamento já exposto nesta discussão que para haver interação, dinamização e motivação

é imperativo o amplo conhecimento da proposta pedagógica do curso ofertado, pois o material didático precisa ser o condutor de um conjunto de atividades que procure levar à construção do conhecimento; daí a necessidade desse material se apresentar em linguagem dialógica que, na ausência física do professor, possa garantir ... em alguns casos, uma conversa entre professor e aluno, tornando sua leitura leve e motivadora (Belisário, 2011, p. 138).

É sabido que "os indivíduos toleram cada vez menos seguir cursos uniformes ou rígidos que não correspondem as suas necessidades reais e à especificidade de seu trajeto de vida" (Lévy, 2001, P.169). Nesta perspectiva, surgem as ofertas de cursos que disponibilizam através de um design atrativo e hipermídias interativas, elementos de inovação na utilização das tecnologias na educação. No entanto, a tecnologia só será relevante se for apropriada para facilitar o cumprimento dos objetivos propostos. Pois, as técnicas por si só não se justificam, mas somente pelo alcance dos objetivos: no caso, a aprendizagem (Masetto, 2006, p.144). Entendemos que "no processo de aprendizagem, só aprende verdadeiramente aquele que se apropria do aprendido, transformando-o em apreendido, com o que pode, por isto mesmo, reinventá-lo; aquele que é capaz de aplicar o apreendido a situações existenciais concretas" (Freire, 1996, p. 16). Outros autores entendem por aprendizagem autônoma aquela que se verifica quando o próprio estudante é gestor do seu processo de aprendizagem, sendo capaz de auto-regular e auto gerir este processo e a capacidade de leitura crítica das informações. Para realmente 
funcionar no sentido de tornar os sujeitos cognoscentes estimulados a se expressar e desenvolver esta autonomia, o processo deve ter como base os diálogos entre professores e estudantes, estudantes e seus pares, estudantes e professores e seus contextos sociais. A todo processo educacional está subjacente os diálogos trocados entre os sujeitos, sejam estes em ambiente presencial ou virtual (Beloni, 1999; Kenski, 2003; Almeida, 2003; Moran, 2000).

De acordo com Beloni (1999, p. 48)

o diálogo deve ser encorajado através de materiais de cursos que ofereçam aos estudantes conhecimentos, habilidades e valores que sejam relevantes para seus interesses e necessidades, e que eles possam usar ativamente para entender, gerir e mudar seus mundos sociais através do diálogo com seus pares.

Nesta perspectiva, buscamos identificar quais as estratégias que são desenvolvidas pelos discentes no ambiente virtual de aprendizagem para a construção de conhecimento; a partir das novas relações com o saber surgido com o movimento da cibercultura, onde uma nova geração nativa digital já é habituada a um volume sufocante de informações e a uma imensa variedade de ferramentas. Mas, apesar da familiaridade com o número excessivo de informações e de ferramentas, esta geração digital chega à sala de aula de instituições de ensino superior com o desafio de saber utilizar criticamente a informação e construir conhecimento. Pois,

a tentação da cópia no mundo da nova mídia é enorme, porque a profusão de informação atinge patamares de verdadeira inundação incontrolável. Por conta disso, muitos alunos que imaginam "pesquisar" na internet não vão além de acumular pedaços de textos ou imagens, tendo como trabalho apenas navegar para coletar dados, sem se preocupar em tornar-se sujeito de conhecimento reconstruído (Demo, 2011, p. 81).

Corroboramos com o pensamento de que o indivíduo constrói o conhecimento a partir de suas interações, e este conhecimento (re) construído distingue-se da mera informação pelo fato de estar vinculado à intencionalidade de quem espera conhecer (Maturana \& Varela, 1995).

\section{CONSIDERAÇÕES FINAIS}

As propostas pedagógicas ativas constituem uma ferramenta de estímulo à aprendizagem inserindo o educando no paradigma educacional contemporâneo, onde os sujeitos vivenciam mudanças nas formas de pensar, de aprender e de se relacionar numa sociedade tecnológica em que as informações se renovam a uma velocidade cada vez mais intensa. Neste cenário, posturas passivas de aprendizagem não mais correspondem aos desafios colocados aos profissionais do século XXI.

$\mathrm{Na}$ área médica a velocidade de atualização das informações e dos conhecimentos científicos é ainda mais rápida. Estima-se que os conhecimentos adquiridos pelo estudante ao longo da sua graduação tornam-se defasados no final dela: "40 a 50\% dos conhecimentos são abandonados ou colocados em dúvida em 4 a 5 anos, levando o médico a uma formação continuada, sempre" (Tsuji, 2010, p. 1).

Salientamos a importância no refinamento das estratégias utilizadas no processo de construção de conhecimento para que estes estudantes possam se reprogramar, dar conta e 
superar os desafios. Pois quanto maior a capacidade de auto gerir seu processo de aprendizagem mais estarão aptos a enfrentar as mudanças. A oferta dos cursos em EAD online, pela sua flexibilidade de tempo e de espaço, pode possibilitar, através dos recursos de hipermídias, a dinamização e otimização dos processos de ensino e aprendizagem, fazendo com que os indivíduos construam conhecimento com autonomia através da estruturação de estratégias que proporcionem a construção de novos e mais complexos conhecimentos.

\section{REFERÊNCIAS}

Almeida, M. E. B. (2003).Educação a distância na internet: abordagens e contribuições dos ambientes digitais de aprendizagem. Educação e Pesquisa, 29(2), 327 - 340.

Alves, L., Barros, D., \& Okada, A. (2009). (Org.) Moodle: Estratégias Pedagógicas e Estudos de Caso. Salvador: EDUNEB.

Anastasiou, L. das C. (2007). Ensinar, aprender, apreender e processos de ensinagem. In: Anastasiou, L C., \& Alves, L.P. (Orgs). Processos de ensinagem na universidade: pressupostos para as estratégias de trabalho em aula. Joinville (SC): UNIVILLE. p.15-43.

Andrade, A. F. de, \& Vicari, R.M. (2011). Construindo um ambiente de aprendizagem a distância inspirado na concepção sociointeracionista de Vygotsky. In: Silva, M. (Org.). Educação online. São Paulo: Loyola. p. $257-293$.

Belisário, A.O. (2011). Material didático na educação à distância e a constituição de propostas interativas. In: Silva, Marco (Org). Educação online. São Paulo: Loyola. p. 135-146.

Bellonni, M.L. (1999). Educação à distância. Campinas: Autores Associados.

Beluce, A.C., \& Oliveira, K.L. (2012). Ambientes virtuais de aprendizagem: das estratégias de ensino às estratégias de aprendizagem. Rio Grande do Sul: Anped.

Borochovicius, E., \& Tortella, J.C.B. (2014). Aprendizagem Baseada em Problemas: um método de ensino-aprendizagem e suas práticas educativas. Ensaio: Aval.Pol.Públ.Educ., 22(83), 263294, Jun.

Castells, M. (2013). O poder da comunicação. Lisboa: Calouste Gulbenkian.

Cyrino, E. G., \& Toralles-Pereira, M. L. (2004). Trabalhando com estratégias de ensinoaprendizado por descoberta na área da saúde: a problematização e a aprendizagem baseada em problemas. Cadernos de Saúde Pública, 20(3), 780-788.

Delors, J. et al. (1998). Educação: um tesouro a descobrir. São Paulo: Cortez.

Dewey, J. (1979). Como Pensamos. São Paulo: Cia Editora Nacional.

Dewey J.(2004). Democracia y educación. Madrid: Morata.

Ferreira Filho, O.F., et al. (2002). Visão docente do processo de implementação da aprendizagem baseada em problemas (ABP) no curso médico da universidade estadual de londrina (UEL). Rev. Bras. Educ. Med. 26(3), 175-183, set./dez. 
Ferreira, J.C. (2008). O Design na Era da Aprendizagem Virtual: para um estudo do design de plataformas de E-learning. Dissertação de Mestrado. Covilhã: UBI.

Freire, P. (1996). Pedagogia da autonomia: saberes necessários à prática educativa. São Paulo: Paz e Terra.

Habermas, J. (2004). Verdade e justificação: ensaios filosóficos. São Paulo: Edições Loyola.

Kenski, V. (2003). Aprendizagem mediada pela tecnologia. Revista Diálogo Educacional, 4(10), p.47-56.

Kuri, N.P., Manzato, G. G., \& Silva, A. N. da. (2004). Aprendizado baseado em problemas em uma plataforma de ensino a distância: Uma aplicação na eesc-usp. Minerva, São Carlos (SP), 4(1), 27-39.

Lévy, P. (2003) Cibercultura. São Paulo: Editora 34.

Masetto, M.T. (2006). Mediação pedagógica e o uso da tecnologia. In: Moran, J. M., Masetto, M. T. \& Behrens, M. A. Novas tecnologias e mediação pedagógica. Campinas: Papirus. p. $133-$ 173.

Maturana, H., \& Varela, F. (1995). A árvore do conhecimento: as bases biológicas do entendimento humano. Campinas: Psy II.

Mehkecke, Q., et al. (2006). Estratégia de interação on-line: um estudo no contexto de falantes da língua portuguesa de Portugal. Novas Tecnologias da Educação, Porto Alegre, 4(1), 1-11.

Melo, M.C.M. (2012). O uso da informação para solução de casos clínicos: investigando uma rede de diálogos presenciais e virtuais. Dissertação de Mestrado, Centro de Educação, UFPE. Recife: UFPE.

Mezzari, A. (2011). O uso da aprendizagem baseada em problemas (ABP) como reforço ao ensino presencial utilizando o ambiente de aprendizagem moodle. Rev. Bras.de Educação Médica, 35(1), 114-121.

Moran, J. M. (1997). Como utilizar a internet na educação. Revista Ciência da Informação, 26(2), 146-153.

Moran, J.M. (1995). Novas tecnologias e o re-encantamento do mundo. Tecnologia Educacional, Rio de Janeiro, 23(126), 24-26, set./out.

Moran, J.M., Masetto, M. T., \& Behrens, M.A. (2000). Novas tecnologias e mediação pedagógica. Campinas: Papirus..

Palloff, M.R., \& Pratt, K. (2002). Construindo comunidades de aprendizagem no ciberespaço: Estratégias eficientes para salas de aula online. Porto Alegre: Artmed.

Perassinoto, M.G.M., Boruchovitch, E., \& Bzuneck, J.A. (2013). Estratégias de aprendizagem e motivação para aprender de alunos do Ensino Fundamental. Avaliação Psicológica, 12(3), 351-359.

Schalch, C.S., \& Melo, M.C.M. (2015). A utilização de recursos de mídias no processo de ensino e aprendizagem: identificação do perfil dos docentes. Rev. Humanitas, 4(1), 28-45, set. 
Schwartz, S.( 2008). Aprendizagem: questão de ritmo? Porto Alegre: EDIPUCRS.

Soares, M.A. (2008). A aplicação do método de ensino problem based learning no curso de ciências. Dissertação Mestrado. São Paulo: USP.

Tostes, S. C. (2011). Estratégias mediadoras no ambiente virtual. Revista Brasileira de Lingüística Aplicada, 11, 1.

Tsuji, H. (2010). Aprendizagem baseada em problemas no curso de medicina. Revista Eletrônica de Jornalismo Científico, EMB.

Vigotsky, L. (2000). A formação social da mente: o desenvolvimento dos processos mentais superiores. São Paulo: Martins Fontes.

Vigotsky, L. (2008). Pensamento e linguagem. São Paulo: Martins Fontes. 